\title{
Association Between Interpregnancy Interval and Risk of Preterm Birth and Its Modification by Folate Intake: The Japan Environment and Children's Study
}

\author{
Kanami Tanigawa ${ }^{1}$, Satoyo Ikehara ${ }^{1}$, Meishan $\mathrm{Cui}^{1}$, Yoko Kawanishi ${ }^{2}$, Tadashi Kimura ${ }^{2}$, Kimiko Ueda ${ }^{3}$,
} Kazumasa Yamagishi ${ }^{4}$, and Hiroyasu Iso ${ }^{1,4}$, for the Japan Environment and Children's Study Group

${ }^{1}$ Public Health, Department of Social Medicine, Osaka University Graduate School of Medicine, Osaka, Japan

${ }^{2}$ Department of Obstetrics and Gynecology, Osaka University Graduate School of Medicine, Osaka, Japan

${ }^{3}$ Osaka Maternal and Child Health Information Center, Osaka Women's and Children's Hospital, Osaka, Japan

${ }^{4}$ Department of Public Health Medicine, Faculty of Medicine, and Health Services Research and Development Center, University of Tsukuba, Ibaraki, Japan

Received January 26, 2021; accepted April 30, 2021; released online May 22, 2021

\begin{abstract}
Background: Both short and long interpregnancy intervals (IPIs) have been associated with risk of preterm birth, but the evidence is limited in Asians. It is also uncertain whether the association is modified by dietary folate intake or folic acid supplementation during pregnancy. Thus, we examined associations between IPI and risk of preterm birth and effect modification of those associations by dietary intake of folate and supplementation with folic acid on the basis of a nationwide birth cohort study.

Methods: Among 103,062 pregnancies registered in the Japan Environment and Children's Study, 55,203 singleton live-birth pregnancies were included in the analysis. We calculated IPI using birth date, gestational age at birth of offspring, and birth data of the latest offspring. Odds ratios (ORs) and 95\% confidence intervals (CIs) of the risk of preterm birth were estimated according to IPI categories.

Results: Both $<6$-month and $\geq 120$-month IPIs were associated with an increased risk of preterm birth, compared with an 18-23month IPI. The multivariable ORs were 1.63 (95\% CI, 1.30-2.04) for $<6$-month and 1.41 (95\% CI, 1.11-1.79) for $\geq 120$-month IPIs. These associations were confined to women with inadequate intake of dietary folate and folic acid supplementation during pregnancy. Multivariable ORs were 1.76 (95\% CI, 1.35-2.29) for <6-month IPI and 1.65 (95\% CI, 1.24-2.19) for $\geq 120$-month IPI.

Conclusion: Both $<6$-month and $\geq 120$-month IPIs were associated with an increased risk of preterm birth. These higher risks were confined to women with inadequate intake of dietary folate and folic acid supplementation during pregnancy.
\end{abstract}

Key words: interpregnancy interval; preterm birth; folate; folic acid; prospective studies

Copyright $\odot 2021$ Kanami Tanigawa et al. This is an open access article distributed under the terms of Creative Commons Attribution License, which permits unrestricted use, distribution, and reproduction in any medium, provided the original author and source are credited.

\section{INTRODUCTION}

Preterm birth is recognized as a worldwide public health problem in terms of survival and quality of life. The World Health Organization (WHO) reported that approximately 15 million infants were born preterm in 2014. ${ }^{1}$ In addition, 1 million children under 5 years old died related to preterm birth complications in $2015 .^{2}$

Interpregnancy interval (IPI) is defined as the interval between the birth date of the latest delivery and conception of the next pregnancy. ${ }^{3}$ Both short and long IPIs have been associated with increased risks for preterm birth in the previous studies reported from the United States, Latin America, and Europe, ${ }^{4-8}$ but the evidence is limited in Asians. Because IPI has become shorter rapidly in Japan since the early $2000 \mathrm{~s},{ }^{9}$ there is a need to consider this association. Nevertheless, there is only one report of the association between IPI and preterm birth in Japanese women. ${ }^{10}$
In that retrospective cohort study of 547 women with a previous history of preterm birth, an IPI of $<12$ months was associated with 2.1-fold higher risk of recurrent preterm birth than that with an IPI $\geq 12$ months. ${ }^{10}$

There is limited evidence regarding modification of risk of preterm birth related to IPI; however, dietary intake of folate and supplementation with folic acid during pregnancy may be potential protective factors for perinatal outcomes related to IPI. ${ }^{11}$ A short IPI without folic acid supplementation was associated with a lower birthweight and higher risk of small size for gestational age ${ }^{12}$; however, it remains uncertain as to whether the association between IPI and risk of preterm birth is modified by dietary intake of folate or supplementation with folic acid during pregnancy. Therefore, we evaluated the associations of IPI with risk of preterm birth and the modification of this association by dietary intake of folate and supplementation with folic acid in a large birth cohort study from Japan. 


\section{METHODS}

\section{Study population}

The Japan Environment and Children's Study (JECS) is a nationwide birth cohort study funded by the Ministry of the Environment, Japan. Pregnant women were recruited between January 2011 and March 2014 from medical facilities or local municipal offices. A total of 103,062 pregnancies were confirmed. The details of the study are described elsewhere. ${ }^{13,14}$ The protocol of this study was approved by the Ministry of the Environment's Institutional Review Board on Epidemiological Studies (date of approval: August 92010 and approval number: 2010-2R) and by the Ethics Committees of all participating institutions. The JECS obtained written informed consent from all participants.

Among 98,255 singleton livebirth pregnancies, we excluded 7 individuals with missing maternal age, 38,586 individuals because of primiparity, 4,045 individuals with missing birth history, and 414 individuals who had uncertain information regarding IPI. Finally, 55,203 singleton livebirth pregnancies were included in the present analysis (Figure 1).

\section{Data collection}

We distributed self-administered questionnaires at registration and during the second/third trimester. Questions included maternal demographic characteristics, socioeconomic status, lifestyle, mental health, and birth date of siblings. Data on the history of use of anemia medication before and during pregnancy were obtained during interviews at registration and during the second/third trimester. Maternal anthropometric data before and during pregnancy and data on complications before and during pregnancy, maternal age at delivery, number of births, birth dates of offspring, gestational age, and perinatal outcomes were obtained from medical records provided by the participants' obstetricians. Body mass index (BMI) before pregnancy was calculated as weight before pregnancy $(\mathrm{kg}) /$ height $^{2}\left(\mathrm{~m}^{2}\right)$.

\section{Exposure and outcome}

IPI is the interval between the birth date of the latest delivery and conception of the next pregnancy. ${ }^{3}$ We defined IPI as the interval between birth date of the latest sibling and the conception of pregnancy registered in the JECS. We converted gestational age from weeks into months and calculated IPI by subtracting the gestational age (in months) at birth of the offspring registered in the JECS from the interval between the date of the two deliveries (in months). ${ }^{3}$ IPI was categorized as <6, 6-11, 12-17, 18-23, 24-29, 30-35, 36-41, 42-47, 48-53, 54-59, 60-89, 90-119, or $\geq 120$ months.

Preterm birth was defined as delivery at $<37$ weeks of gestation, based on medical record transcripts. Gestational age was estimated clinically from the date of last menstrual period, by measuring the crown-rump length, or from the date of in vitro fertilization.

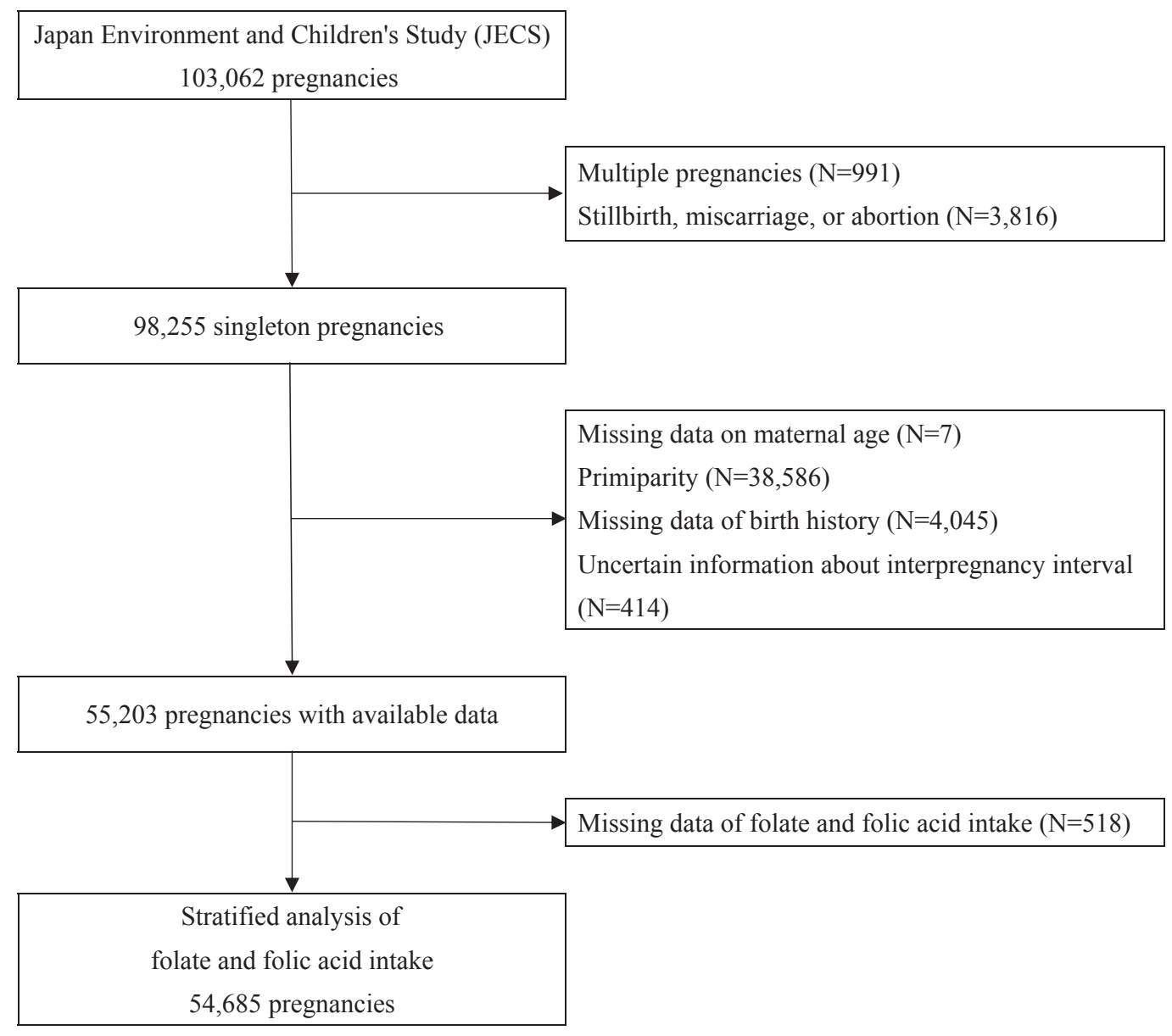

Figure 1. Flow chart of participant selection. 


\section{Intake of dietary folate and folic acid supplementa- tion}

Dietary intake of folate during pregnancy was calculated from the food frequency questionnaire (FFQ) ${ }^{15}$ administered during the second/third trimester. In the FFQ, we estimated daily dietary intake by multiplying frequency by the standard portion size of food items, on the basis of the Japanese Food Composition Tables, $5^{\text {th }}$ Revision. ${ }^{16}$ Spearman's correlation coefficient comparing FFQ and 12-day food records over four seasons at intervals of each 3 months was 0.62 for dietary intake of folate. ${ }^{15}$

Information about supplementation with folic acid during pregnancy was included in the questionnaire and in FFQ administered during the second/third trimester. In that questionnaire, we asked about the frequency of supplementation with folic acid: $\geq 2$ times/day, once/day, 4-6 times/week, 1-3 times/week, 2-3 times/month, once/month, or never. In the FFQ administered during the second/third trimester, if participants used supplements during pregnancy, they wrote down the trade names or types of supplements used and selected the category for the frequency of use of each supplement: $\geq 4$ times/day, 2-3 times/day, once/day, 5-6 times/week, 3-4 times/week, or 1-2 times/week. When participants answered the frequency of folic acid supplementation as once or more times/day in the questionnaire or FFQ during the second/third trimester, we defined these cases as daily supplementation of folic acid.

Furthermore, we defined dietary intake of $\geq 400 \mu \mathrm{g}$ /day of folate and/or daily supplementation with folic acid as adequate intake during pregnancy. We defined folate dietary intake $<400 \mu \mathrm{g} /$ day and no daily supplementation of folic acid as inadequate intake during pregnancy. This is because Japanese pregnant women are recommended to take at least $400 \mu \mathrm{g}$ /day folate as part of their diet during the second/third trimester, ${ }^{17}$ and most folic acid supplements in Japan contain $\geq 400 \mu \mathrm{g} /$ day folic acid. ${ }^{18}$ Bioavailability of folate is 50 percent lower than that of folic acid. ${ }^{19}$

\section{Statistical analyses}

The present analysis is based on the dataset jecs-an-20180131, which was released in March 2018. We calculated means and prevalence of characteristics according to IPI. Test for trends were performed modeling with the median value in each category of IPI. Multivariable logistic regression models were used to calculate the maternal age-adjusted and multivariable odds ratios (ORs) and their corresponding 95\% confidence intervals (CIs) for the association between IPI and the risk of preterm birth. The reference category of IPI was defined as 18-23 months, because WHO recommends that the next pregnancy be avoided for at least 18 months from birth. ${ }^{20}$ In the multivariable model, we adjusted for maternal age at delivery (continuous) and potential confounders including residential area (15 regional centers), maternal BMI before pregnancy (quintile), maternal education levels (junior high school, high school, technical college/vocational school/junior college, or university/graduate school), maternal smoking during pregnancy (non-smoker, ex-smoker, or current smoker), frequency of maternal passive smoking during pregnancy (almost never, once, 2-6 days, or every day per week), maternal drinking during pregnancy (non-drinker, exdrinker, or current drinker), maternal physical activity during pregnancy (The short version of the International Physical Activity Questionnaire [IPAQ] ${ }^{21,22}$; quintiles), household income during pregnancy $(<2,2-3.9,4-5.9,6-7.9$, or $\geq 8$ million yen/ year), maternal occupation during pregnancy (full-time worker, self-employed worker, dispatched worker, part-time worker, housewife, or other), marital status during pregnancy (married, unmarried, or divorced/widowed), number of children (1, 2, or $\geq 3$ children), maternal stress during pregnancy (Kessler Psychological Distress Scale $[\mathrm{K} 6]^{23}:<5,5-12$, or $\geq 13$ points), maternal dietary intake of iron during pregnancy (quintiles), maternal use of anemia medication during pregnancy (yes or no), maternal dietary intake of folate and supplementation with folic acid during pregnancy (inadequate intake of dietary folate and folic acid supplementation during pregnancy or adequate intake of dietary folate and/or folic acid supplementation during pregnancy), the latest pregnancy outcome (livebirth or stillbirth/ miscarriage/abortion), spontaneous pregnancy (yes or no), previous history of preterm birth (yes or no), maternal medical history of hypertensive disorder of pregnancy (yes or no), and gestational diabetes (yes or no). Missing data for confounding factors were included as categorical variables in the model. The proportions of missing values were $7.4 \%$ for household income during pregnancy, $6.4 \%$ for previous history of preterm birth, $5.8 \%$ for maternal physical activity during pregnancy, and 0 to $1.8 \%$ for other confounding factors.

We measured the interaction between IPI and folate/folic acid intake on the risk of preterm birth, and a stratified analysis was conducted according to folate and/or folic acid intake. We divided dietary intake of folate and supplementation with folic acid during pregnancy into two categories (adequate or inadequate) $(N=54,685)$.

All statistical tests were two-tailed, and $P<0.05$ was considered statistically significant. All analyses were conducted using SAS software version 9.4 (SAS Institute, Inc., Cary, NC, USA).

\section{RESULTS}

Table 1 shows the baseline characteristics of the participants in terms of IPI; $2.9 \%$ of women had <6-month IPIs and $2.4 \%$ had $\geq 120$-month IPIs. Compared to those women with $<6$-month IPI, those with longer IPIs were older, were younger at first birth, reported less physical activity during pregnancy, and had earlier gestational age at birth. They were more likely to have $\geq 2$ children, to use anemia medication during pregnancy, to report stillbirth/miscarriage/abortion of the last pregnancy, to have hypertensive disorder of pregnancy and gestational diabetes, and to deliver a baby with birthweight $<2,500 \mathrm{~g}$. They had higher household income, were less likely to have a job during pregnancy, have a spouse during pregnancy, and have a spontaneous pregnancy.

Table 2 shows the maternal age-adjusted and multivariable ORs and 95\% CIs for preterm birth. Among 55,203 pregnancies, 521 early preterm births ( 22 to $<34$ weeks of gestation) and 2,094 late preterm births ( 34 to $<37$ weeks of gestation) were identified from medical record transcripts. Compared with women who had 18-23-month IPIs, both $<6$-month and $\geq 120$-month IPIs were associated with increased risk of preterm birth. The multivariable ORs were 1.63 (95\% CI, 1.30-2.04) for <6-month IPI and 1.41 (95\% CI, 1.11-1.79) for $\geq 120$-month IPI.

Table 3 displays the maternal age-adjusted and multivariable ORs and $95 \%$ CIs for preterm birth stratified by dietary intake of folate and supplementation with folic acid during pregnancy. The 
Table 1. Mean and prevalence of characteristics according to interpregnancy interval in the 55,203 pregnancies

\begin{tabular}{|c|c|c|c|c|c|c|c|c|c|c|c|c|c|c|}
\hline & \multicolumn{13}{|c|}{ Interpregnancy interval (months) } & \multirow{2}{*}{$P$ for trend } \\
\hline & $<6$ & $6-11$ & $12-17$ & $18-23$ & $24-29$ & $30-35$ & $36-41$ & $42-47$ & $48-53$ & $54-59$ & $60-89$ & $90-119$ & $\geq 120$ & \\
\hline Participants, $n$ & 1,587 & 5,349 & 9,388 & 8,526 & 6,721 & 4,903 & 3,901 & 2,967 & 2,257 & 1,807 & 4,724 & 1,755 & 1,318 & \\
\hline Age at delivery, year & 28.5 & 29.7 & 30.7 & 31.4 & 32.1 & 32.5 & 32.9 & 33.1 & 33.6 & 33.8 & 34.3 & 35.3 & 37.0 & $<0.001$ \\
\hline Age at first birth, year & 25.2 & 26.7 & 27.4 & 27.7 & 27.9 & 27.8 & 27.7 & 27.3 & 27.3 & 26.9 & 26.1 & 24.6 & 23.1 & $<0.001$ \\
\hline BMI before pregnancy, $\mathrm{kg} / \mathrm{m}^{2}$ & 22.2 & 21.4 & 21.1 & 21.1 & 21.2 & 21.3 & 21.5 & 21.5 & 21.4 & 21.6 & 21.7 & 22.0 & 22.1 & $<0.001$ \\
\hline College or higher education, $\%$ & 9.7 & 17.2 & 21.7 & 23.2 & 22.7 & 23.7 & 21.3 & 19.2 & 17.7 & 18.5 & 15.1 & 9.4 & 5.1 & $<0.001$ \\
\hline Smoking during pregnancy, $\%$ & 11.9 & 6.2 & 3.9 & 3.5 & 3.8 & 4.0 & 4.0 & 4.9 & 5.2 & 5.3 & 7.3 & 10.0 & 15.8 & $<0.001$ \\
\hline Passive smoking during pregnancy: $\geq 1$ time/week, $\%$ & 49.2 & 39.7 & 33.4 & 33.2 & 34.0 & 33.6 & 35.7 & 36.8 & 37.7 & 37.9 & 42.0 & 45.4 & 51.7 & $<0.001$ \\
\hline Drinking during pregnancy, $\%$ & 4.4 & 4.1 & 4.0 & 3.7 & 3.6 & 3.7 & 3.6 & 3.6 & 3.5 & 3.8 & 3.9 & 4.2 & 5.2 & 0.173 \\
\hline Physical activity during pregnancy, METs*min/day & 307 & 283 & 272 & 249 & 245 & 228 & 225 & 214 & 209 & 215 & 210 & 237 & 270 & $<0.001$ \\
\hline Household income: $<4$ million yen/year, $\%$ & 57.9 & 50.4 & 42.8 & 38.8 & 36.6 & 35.7 & 36.0 & 36.4 & 34.9 & 37.9 & 38.0 & 41.2 & 46.1 & $<0.001$ \\
\hline Having job during pregnancy, $\%$ & 63.9 & 58.9 & 56.7 & 52.7 & 49.4 & 49.0 & 47.4 & 46.5 & 43.8 & 43.3 & 44.1 & 44.0 & 36.7 & $<0.001$ \\
\hline Having spouse during pregnancy, $\%$ & 99.0 & 99.1 & 99.4 & 99.4 & 99.1 & 99.0 & 98.8 & 98.6 & 98.8 & 97.4 & 96.6 & 94.1 & 89.0 & $<0.001$ \\
\hline Number of children: $\geq 2$ children, $\%$ & 37.4 & 32.6 & 29.7 & 30.8 & 31.4 & 33.1 & 34.0 & 37.6 & 37.9 & 41.2 & 44.8 & 45.4 & 46.5 & $<0.001$ \\
\hline Stress during pregnancy: $\mathrm{K} 6 \geq 13$ points, $\%$ & 4.8 & 3.6 & 2.5 & 2.8 & 2.6 & 2.8 & 2.4 & 2.8 & 3.3 & 3.9 & 3.5 & 4.2 & 5.1 & $<0.001$ \\
\hline Dietary intake of iron during pregnancy, $\mathrm{mg} /$ day & 6.7 & 6.8 & 6.9 & 6.8 & 7.0 & 6.9 & 6.9 & 6.8 & 6.8 & 6.8 & 6.8 & 6.8 & 6.8 & 0.500 \\
\hline Use of anemia medication during pregnancy, $\%$ & 14.7 & 12.3 & 11.4 & 12.9 & 13.8 & 14.4 & 14.8 & 15.9 & 16.7 & 16.4 & 15.9 & 16.9 & 16.6 & $<0.001$ \\
\hline Adequate intake of folate and/or folic acid, $\%$ & 23.2 & 26.7 & 28.0 & 28.9 & 29.3 & 29.8 & 30.5 & 29.4 & 27.8 & 28.2 & 27.0 & 28.7 & 28.5 & 0.397 \\
\hline Spontaneous pregnancy, $\%$ & 99.4 & 98.7 & 97.6 & 96.6 & 96.2 & 95.1 & 95.4 & 95.3 & 93.8 & 94.2 & 94.6 & 95.3 & 95.4 & $<0.001$ \\
\hline $\begin{array}{l}\text { Pregnancy outcome of last pregnancy: stillbirth, } \\
\text { miscarriage or abortion, } \%\end{array}$ & 4.0 & 4.9 & 6.4 & 10.9 & 14.1 & 18.1 & 19.5 & 22.2 & 21.6 & 26.9 & 27.4 & 32.9 & 38.2 & $<0.001$ \\
\hline Previous history of preterm birth, $\%$ & 5.1 & 5.0 & 5.0 & 5.3 & 5.1 & 6.2 & 5.5 & 6.4 & 6.3 & 7.0 & 7.5 & 8.2 & 6.8 & $<0.001$ \\
\hline Hypertensive disorder of pregnancy, $\%$ & 1.6 & 1.7 & 1.6 & 1.6 & 1.9 & 2.1 & 2.4 & 2.2 & 2.2 & 3.3 & 3.5 & 4.2 & 5.8 & $<0.001$ \\
\hline Gestational diabetes, $\%$ & 2.1 & 2.2 & 2.1 & 2.5 & 2.4 & 2.7 & 3.2 & 2.7 & 3.5 & 3.2 & 3.8 & 4.3 & 4.8 & $<0.001$ \\
\hline Birthweight: $<2,500 \mathrm{~g} \%$ & 8.5 & 6.7 & 5.9 & 6.9 & 7.2 & 7.7 & 7.5 & 7.4 & 7.4 & 7.7 & 8.3 & 9.1 & 11.2 & $<0.001$ \\
\hline Gestational age at birth, weeks & 38.6 & 38.7 & 38.7 & 38.7 & 38.7 & 38.7 & 38.7 & 38.6 & 38.7 & 38.6 & 38.6 & 38.5 & 38.4 & $<0.001$ \\
\hline Early preterm birth ( 22 to $<34$ weeks), $\%$ & 1.8 & 0.8 & 0.6 & 0.8 & 0.8 & 0.9 & 0.8 & 1.1 & 1.0 & 0.8 & 1.1 & 1.8 & 2.2 & $<0.001$ \\
\hline Late preterm birth (34 to <37 weeks), $\%$ & 5.4 & 3.6 & 3.6 & 3.7 & 3.2 & 3.5 & 3.4 & 3.7 & 3.3 & 4.3 & 4.2 & 5.1 & 6.6 & $<0.001$ \\
\hline
\end{tabular}

K6, Kessler Psychological Distress Scale; METs, metabolic equivalents.

$P$ for interaction between IPI and folate/folic acid intake during pregnancy on the risk of preterm birth was 0.38 . Higher risks for preterm birth related to $<6$-month and $\geq 120$-month IPIs compared with 18-23-month IPI were found among women with an inadequate intake of dietary folate and folic acid supplementation, while there was no association among women with an adequate intake of dietary folate and/or folic acid supplementation during pregnancy. The multivariable ORs were 1.76 (95\% CI, 1.35-2.29) for <6-month IPI and 1.65 (95\% CI, 1.24-2.19) for $\geq 120$ month-IPI among women with an inadequate intake of dietary folate and folic acid supplementation during pregnancy, and 1.26 (95\% CI, 0.76-2.10) for <6-month IPI and 0.88 (95\% CI, 0.52-1.47) for $\geq 120$-month IPI among women with an adequate intake of dietary folate and/or folic acid supplementation during pregnancy.

\section{DISCUSSION}

In a large birth cohort study, we found that both $<6$-month and $\geq 120$-month IPIs were associated with increased risk of preterm birth. For $<6$-month and $\geq 120$-month IPIs, the risks of preterm birth were 1.4- to 1.6-fold higher than those with 18-23-month IPI. The interaction between IPI and folate/folic acid intake on the risk of preterm birth was not statistically significant; however, higher risks of preterm birth were confined to women with inadequate intake of dietary folate or those with folic acid supplementation during pregnancy.

Our findings are consistent with those of previous studies., ${ }^{4,5}$ A United States study using vital statistics data among approximately 170,000 singleton livebirths found that, compared with 18-23-month IPI, both $0-5$-month and $\geq 120$-month IPIs were associated with 1.4 - to 1.5 -fold increased risk of preterm birth (livebirths at $<37$ weeks of gestation). ${ }^{4}$ Another United States study using birth certificate data of approximately 430,000 singleton livebirths reported that both $0-5$-month and $\geq 120$ month IPIs were associated with higher risk of preterm birth (live births at $<37$ weeks of gestation), regardless of race; multivariable-adjusted ORs compared with 18-23-month IPIs according to race were 1.3 (95\% CI, 1.2-1.4) for white women with 0-5-month IPI, 1.4 (95\% CI, 1.3-1.5) for white women with $\geq 120$-month IPI, 1.2 (95\% CI, 1.1-1.3) for black women with 0-5-month IPI, and 1.3 (95\% CI, 1.2-1.4) for black women with $\geq 120$-month IPI. ${ }^{5}$ Regarding the Asian population, a Taiwanese retrospective cohort study reported that $<12$-month IPI led to a 1.3-fold increased risk of preterm birth (live birth at 20-36 weeks of gestation) compared with $\geq 12$-month $\mathrm{IPI}^{24}$; however, a detailed analysis of the association between the fine categories of IPI and risk of preterm birth was not conducted.

The observed higher risk of preterm birth associated with short IPI may be explained by the maternal nutritional depletion hypothesis, in which lactation and insufficient time to recover from the physiological stress of the previous pregnancy adversely affect maternal nutritional status. ${ }^{25,26}$ Maternal folate concentrations remained lower after delivery, and $20 \%$ of women had folate deficiency 6 months after delivery. ${ }^{11}$ Pregnant women with short IPI have an increased risk of folate deficiency - a risk factor for preterm birth. ${ }^{27-29}$

Nevertheless, it is unclear why a long IPI increased the risk of preterm birth. Zhu et al offered two hypotheses about the mechanisms for this. ${ }^{4}$ The first is the "physiological regression hypothesis," by which pregnancy causes anatomical, physiological, and biochemical adaptations in the reproductive system that gradually return to baseline conditions after delivery. When women are not pregnant or have not delivered a baby for a long 

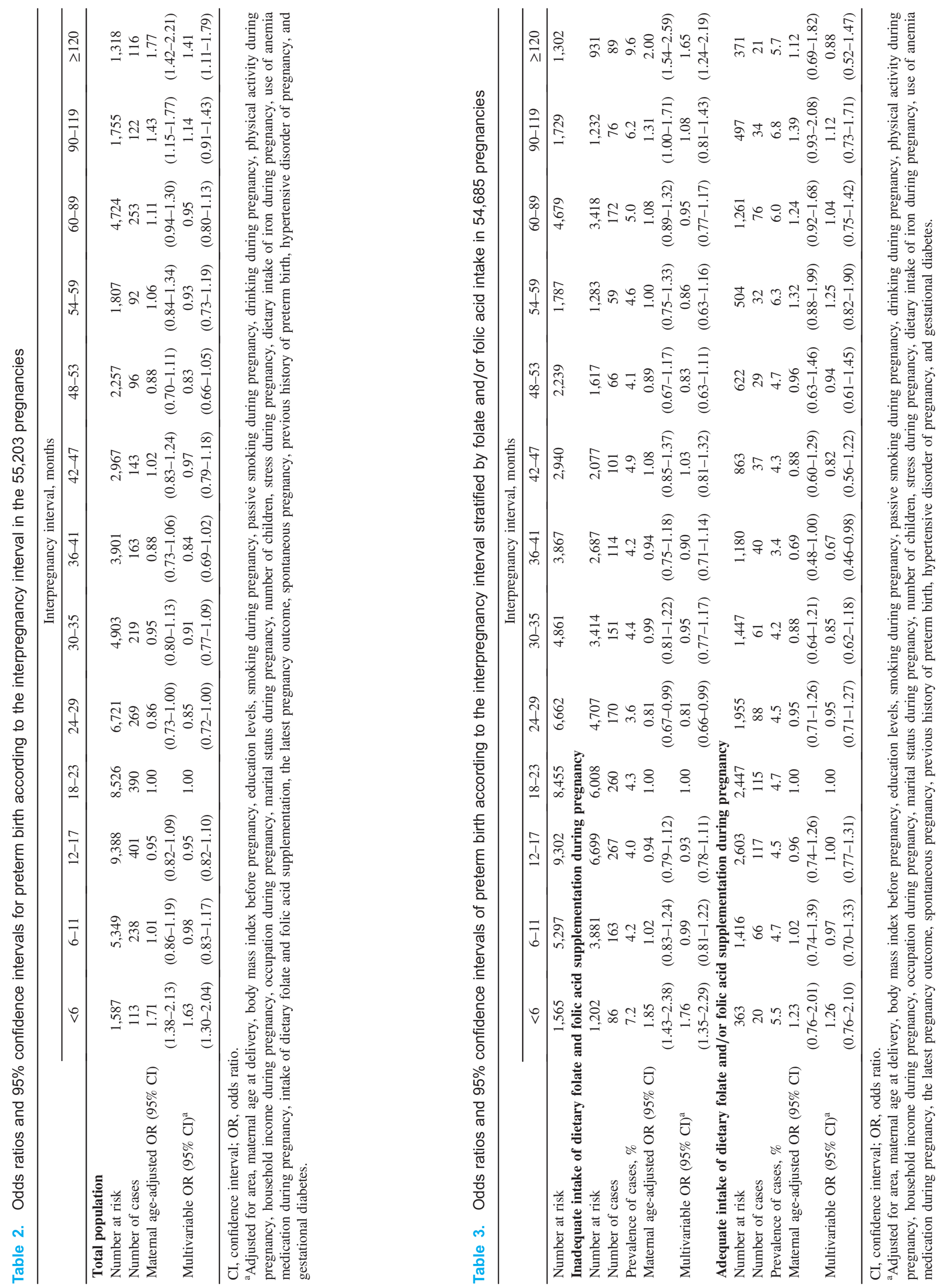
time, the characteristics of reproductive system may become similar to those of women who become pregnant for the first time. The second hypothesis is that a long IPI may be caused by infertility-related factors, thereby increasing the risk of preterm birth. ${ }^{30}$

We found a higher risk of preterm birth related to $<6$-month and $\geq 120$-month IPIs among women with inadequate intake of dietary folate and folic acid supplementation during pregnancy. Folate is a cofactor for single-carbon transfers in the metabolism of nucleotides and amino acids; folate deficiency caused higher homocysteine levels, ${ }^{31-34}$ which is a risk factor for atherosclerosis $^{35}$ and thrombosis. ${ }^{36,37}$ For pregnant women, lower folate levels might affect placentation during pregnancy. A previous study reported that pregnant rats without folate supplementation tended to have lower plasma folate levels, higher plasma homocysteine levels, and decreased placental DNA methylation and placental weight. ${ }^{38}$ For pregnant women, lower blood folate levels $(\leq 9.2 \mathrm{nmol} / \mathrm{L})$ during pregnancy (median: 13.2 weeks of gestation) were associated with lower placental weights and higher risks of preterm birth ( $<37$ weeks of gestation) ${ }^{27}$

The strengths of our study include its prospective design and the large sample size in a national birth cohort. We examined associations between fine categories of IPI and risk of preterm birth after extensive adjustment for potentially confounding factors.

Our study has a few limitations that need mentioning. First, we could not consider pregnancy following miscarriage to calculate IPI. A recent meta-analysis of ten studies indicated that a $<6$ month IPI following miscarriage was not associated with a higher risk of preterm birth. ${ }^{39}$ Second, information regarding supplementation with folic acid during pregnancy was collected from self-reported questionnaires. Nevertheless, a previous study reported that Pearson's correlation coefficient for comparing questionnaire data with interview responses was 0.76 for supplementation with folic acid. ${ }^{40}$ Third, due to the lack of information on presence or absence of labor, which is necessary to define spontaneous preterm birth ${ }^{41}$ we could not discriminate preterm birth as spontaneous or indicated preterm birth. However, a previous study showed that $<6$-month IPI was associated with an increased risk of both spontaneous and indicated preterm birth ( $<37$ weeks of gestation) than was 18 -month IPI. ${ }^{42}$

In conclusion, both $<6$-month and $\geq 120$-month IPIs were associated with increased risk of preterm birth in pregnant Japanese women. The interaction between IPI and folate/folic acid intake on risk of preterm birth was not statistically significant; however, higher risks of preterm birth related to $<6$-month and $\geq 120$-month IPIs were found in women with inadequate intake of dietary folate and folic acid supplementation during pregnancy, suggesting that adequate intake of dietary folate or folic acid supplementation during pregnancy may contribute to decreased risk of preterm birth related to IPI.

\section{ACKNOWLEDGEMENTS}

We appreciate all concerned and the participants. The findings and conclusions of this paper are solely the responsibility of the authors and do not represent official views of the above government.

Members of the JECS Group as of 2021: Michihiro Kamijima (principal investigator, Nagoya City University, Nagoya, Japan), Shin Yamazaki (National Institute for Environmental Studies,
Tsukuba, Japan), Yukihiro Ohya (National Center for Child Health and Development, Tokyo, Japan), Reiko Kishi (Hokkaido University, Sapporo, Japan), Nobuo Yaegashi (Tohoku University, Sendai, Japan), Koichi Hashimoto (Fukushima Medical University, Fukushima, Japan), Chisato Mori (Chiba University, Chiba, Japan), Shuichi Ito (Yokohama City University, Yokohama, Japan), Zentaro Yamagata (University of Yamanashi, Chuo, Japan), Hidekuni Inadera (University of Toyama, Toyama, Japan), Takeo Nakayama (Kyoto University, Kyoto, Japan), Hiroyasu Iso (Osaka University, Suita, Japan), Masayuki Shima (Hyogo College of Medicine, Nishinomiya, Japan), Hiroshige Nakamura (Tottori University, Yonago, Japan), Narufumi Suganuma (Kochi University, Nankoku, Japan), Koichi Kusuhara (University of Occupational and Environmental Health, Kitakyushu, Japan), and Takahiko Katoh (Kumamoto University, Kumamoto, Japan).

Funding sources: Ministry of the Environment, Japan.

Authors' contribution: KT and HI designed the research. SI, TK, KU and HI collected data. KT conducted data analysis. $\mathrm{KT}$, SI, MC, YK, KY and HI contributed to data interpretation. KT drafted the initial manuscript. HI and SI made critical revisions. All authors reviewed and commented upon the manuscript. All authors approved the final manuscript.

Conflicts of interest: None declared.

\section{REFERENCES}

1. Chawanpaiboon S, Vogel JP, Moller AB, et al. Global, regional, and national estimates of levels of preterm birth in 2014: a systematic review and modelling analysis. Lancet Glob Health. 2019;7:e37e46.

2. Liu L, Oza S, Hogan D, et al. Global, regional, and national causes of under-5 mortality in 2000-15: an updated systematic analysis with implications for the Sustainable Development Goals. Lancet. 2016;388:3027-3035.

3. Copen CE, Thoma ME, Kirmeyer S. Interpregnancy intervals in the united states: data from the birth certificate and the national survey of family growth. Natl Vital Stat Rep. 2015;64:1-10.

4. Zhu BP, Rolfs RT, Nangle BE, Horan JM. Effect of the interval between pregnancies on perinatal outcomes. N Engl J Med. 1999; 340:589-594.

5. Zhu BP, Haines KM, Le T, McGrath-Miller K, Boulton ML. Effect of the interval between pregnancies on perinatal outcomes among white and black women. Am J Obstet Gynecol. 2001;185:14031410.

6. Conde-Agudelo A, Rosas-Bermúdez A, Kafury-Goeta AC. Birth spacing and risk of adverse perinatal outcomes: a meta-analysis. JAMA. 2006;295:1809-1823.

7. Fuentes-Afflick E, Hessol NA. Interpregnancy interval and the risk of premature infants. Obstet Gynecol. 2000;95:383-390.

8. Conde-Agudelo A, Belizán JM, Norton MH, Rosas-Bermúdez A. Effect of the interpregnancy interval on perinatal outcomes in Latin America. Obstet Gynecol. 2005;106:359-366.

9. Vital Statistics. Ministry of Health, Labor and Welfare. https:// www.mhlw.go.jp/toukei/list/81-1.html. Published 2017. Accessed May 26, 2019.

10. Yamashita M, Hayashi S, Endo M, et al; Obstetric Research Collaborative in Osaka (ORCO). Incidence and risk factors for recurrent spontaneous preterm birth: a retrospective cohort study in Japan. J Obstet Gynaecol Res. 2015;41:1708-1714.

11. Smits LJ, Essed GG. Short interpregnancy intervals and unfavourable pregnancy outcome: role of folate depletion. Lancet. 2001; 358:2074-2077.

12. van Eijsden M, Smits LJM, van der Wal MF, Bonsel GJ. Association between short interpregnancy intervals and term birth weight: the role of folate depletion. Am J Clin Nutr. 2008;88:147-153. 
13. Kawamoto T, Nitta H, Murata $\mathrm{K}$, et al; Working Group of the Epidemiological Research for Children's Environmental Health. Rationale and study design of the Japan environment and children's study (JECS). BMC Public Health. 2014;14:25.

14. Michikawa T, Nitta H, Nakayama SF, et al; Japan Environment and Children's Study Group. Baseline profile of participants in the Japan Environment and Children's Study (JECS). J Epidemiol. 2018;28: 99-104.

15. Yokoyama Y, Takachi R, Ishihara J, et al. Validity of Short and Long Self-Administered Food Frequency Questionnaires in Ranking Dietary Intake in Middle-Aged and Elderly Japanese in the Japan Public Health Center-Based Prospective Study for the Next Generation (JPHC-NEXT) Protocol Area. J Epidemiol. 2016;26: 420-432.

16. Science and Technology Agency. Standard Tables of Food Composition in Japan. The Fifth Revised Edition. Tokyo; 2000.

17. Dietary reference intakes for Japanese 2020. Ministry of Health, Labour and Welfare. https://www.mhlw.go.jp/stf/seisakunitsuite/ bunya/kenkou_iryou/kenkou/eiyou/syokuji_kijyun.html. Accessed January $23,2020$.

18. Health food to take folic acid, which contribute to fetal development. National Consumer Affairs Center of Japan. http://www.kokusen. go.jp/news/data/n-20110526_1.html. Accessed March 10, 2021.

19. IOM (Institute of Medicine). Dietary Reference Intakes for Thiamin, Riboflavin, Niacin, Vitamin B6, Folate, Vitamin B12, Pantothenic Acid, Biotin, and Choline. National Academies Press; 1998.

20. WHO. Report of a WHO Technical Consultation on Birth Spacing. Rep a WHO Tech Consult Birth Spacing. 2005;13:1-44.

21. Craig CL, Marshall AL, Sjöström M, et al. International physical activity questionnaire: 12-country reliability and validity. Med Sci Sports Exerc. 2003;35:1381-1395.

22. Murase N, Katsumura $\mathrm{T}$, Ueda $\mathrm{C}$, Inoue $\mathrm{S}$, Shimomitsu $\mathrm{T}$. International standardization of physical activity level: reliability and validity study of the Japanese version of the International Physical Activity Questionnaire (IPAQ). J Heal Welf Stat (Kosei no Shihyo). 2002;49:1-9.

23. Furukawa TA, Kawakami N, Saitoh M, et al. The performance of the Japanese version of the K6 and K10 in the World Mental Health Survey Japan. Int J Methods Psychiatr Res. 2008;17:152-158.

24. Hsieh TT, Chen SF, Shau WY, Hsieh CC, Hsu JJ, Hung TH. The impact of interpregnancy interval and previous preterm birth on the subsequent risk of preterm birth. J Soc Gynecol Investig. 2005;12: 202-207.

25. Miller JE. Birth intervals and perinatal health: an investigation of three hypotheses. Fam Plann Perspect. 1991;23:62-70.

26. Winkvist A, Rasmussen KM, Habicht JP. A new definition of maternal depletion syndrome. Am J Public Health. 1992;82:691694.
27. Bergen NE, Jaddoe VWV, Timmermans S, et al. Homocysteine and folate concentrations in early pregnancy and the risk of adverse pregnancy outcomes: the Generation R Study. BJOG. 2012;119: 739-751.

28. Scholl TO, Hediger ML, Schall JI, Khoo CS, Fischer RL. Dietary and serum folate: their influence on the outcome of pregnancy. Am J Clin Nutr. 1996;63:520-525.

29. Siega-Riz AM, Savitz DA, Zeisel SH, Thorp JM, Herring A. Second trimester folate status and preterm birth. Am J Obstet Gynecol. 2004; 191:1851-1857.

30. Messerlian C, Maclagan L, Basso O. Infertility and the risk of adverse pregnancy outcomes: a systematic review and meta-analysis. Hum Reprod. 2013;28:125-137.

31. Bailey LB, Gregory JF 3rd. Folate metabolism and requirements. J Nutr. 1999;129:779-782.

32. Tamura T, Picciano MF. Folate and human reproduction. Am J Clin Nutr. 2006;83:993-1016.

33. Cetin I, Berti C, Calabrese S. Role of micronutrients in the periconceptional period. Hum Reprod Update. 2010;16:80-95.

34. Jacques PF, Selhub J, Bostom AG, Wilson PW, Rosenberg IH. The effect of folic acid fortification on plasma folate and total homocysteine concentrations. N Engl J Med. 1999;340:1449-1454.

35. Boushey CJ, Beresford SAA, Omenn GS, Motulsky AG. A quantitative assessment of plasma homocysteine as a risk factor for vascular disease: probable benefits of increasing folic acid intakes. JAMA. 1995;274:1049-1057.

36. Graham IM, Daly LE, Refsum HM, et al. Plasma homocysteine as a risk factor for vascular disease. The European Concerted Action Project. JAMA. 1997;277:1775-1781.

37. Perry IJ, Refsum H, Morris RW, Ebrahim SB, Ueland PM, Shaper AG. Prospective study of serum total homocysteine concentration and risk of stroke in middle-aged British men. Lancet. 1995;346: 1395-1398

38. Kim JM, Hong K, Lee JH, Lee S, Chang N. Effect of folate deficiency on placental DNA methylation in hyperhomocysteinemic rats. J Nutr Biochem. 2009;20:172-176.

39. Kangatharan C, Labram S, Bhattacharya S. Interpregnancy interval following miscarriage and adverse pregnancy outcomes: systematic review and meta-analysis. Hum Reprod Update. 2017;23:221-231.

40. Satia-Abouta J, Patterson RE, King IB, et al. Reliability and validity of self-report of vitamin and mineral supplement use in the vitamins and lifestyle study. Am J Epidemiol. 2003;157:944-954.

41. Goldenberg RL, Culhane JF, Iams JD, Romero R. Epidemiology and causes of preterm birth. Lancet. 2008;371:75-84.

42. Schummers L, Hutcheon JA, Hernandez-Diaz S, et al. Association of short interpregnancy interval with pregnancy outcomes according to maternal age. JAMA Intern Med. 2018;178:1661-1670 